\title{
Detection of Nucleoside/Nucleotide Drug Resistant Mutants in Liver Cancer Cases: An Experience from India
}

\author{
Manash Pratim Sarma ${ }^{1,2,3^{*}}$, Giasuddin Ahmed ${ }^{2}$, Subhash Medhi ${ }^{2}$, Dipu Bharali ${ }^{1}$, Minakshi \\ Bhattacharjee $^{3}$ and Premashis Kar ${ }^{1}$ \\ ${ }^{1}$ Department of Medicine, Maulana Azad Medical College, University of Delhi, New Delhi, India; ${ }^{2}$ Department of Biotechnology, Gauhati \\ University, Guwahati, Assam, India; ${ }^{3}$ Department of Biotechnology, Assam Down Town University, Panikhaiti, Guwahati, Assam, India
}

\begin{abstract}
Use of nucleoside and nucleotide analogues to treat patients infected with hepatitis B virus (HBV) has been found to be associated with mutations in the polymerase gene of the virus. The current study was carried out in HBV-related hepatocellular carcinoma (HCC) cases to trace the presence of drug-related mutants. A total of $75 \mathrm{HBV}$-related HCC cases were included for the study as per Bruix et al., 2001 EASL guidelines. HBV viral DNA was isolated by the previously standardized manual phenol-chloroform methods. The $3.2 \mathrm{~kb}$ genome of HBV was amplified by six sets of overlapping primers. The amplicons were sequenced commercially [Macrogen, South Korea (ABI PRISM)]. Sequences for the polymerase gene were analyzed using commercial bioinformatics software (http://www.hepseq.org/Public/Tool/annotator_tool.php). The different drug-resistant mutations detected were confirmed twice, ahead of reporting. Four drug-resistant mutations were detected in total: L80I (lamivudine), N236T (adefovir), I169T (entecavir) and A181V (lamivudine/adefovir). Interestingly, all four of the drug-resistant mutants were found in genotype D of HBV. The low number (only four) of drug-resistant mutations detected in this study population can be attributed to the fact that most of the cases were not treated and presented late. This study's findings confirm the presence of previously reported drug-resistant mutations in the HBV genome infecting Indian patients; however, its associations with late stage disease and with the virus genotype $\mathrm{D}$, in particular, need to be further studied in a larger population.
\end{abstract}

\section{Introduction}

The treatment measures for hepatitis B virus (HBV) that are based upon nucleoside/nucleotide analogues have produced mutations in the HBV polymerase region of the viral genome. Antiviral resistance to lamivudine (LAM) has been reported in the YMDD locus, which lies in the catalytic domain of the HBV polymerase gene. ${ }^{1}$

Keywords: Hepatitis B virus; Hepatocellular carcinoma; Indian population; Drugresistant mutation; Polymerase gene.

Abbreviations: HCC, Hepatocellular Carcinoma; HBV, Hepatitis B Virus; kb, kilo base; rt, reverse transcriptase; Lam, Lamivudine; ENT, Entecavir; ADV, Adefovir.

Received: 21 July 2016; Revised: 11 August 2016; Accepted: 26 August 2016

DOI: 10.14218/ERHM.2016.00008

*Correspondence to: Manash Pratim Sarma, Department of Biotechnology, Assam Down Town University, Panikhaiti, Guwahati, Assam, India. Tel: +91-8255075275, E-mail:manash3268@gmail.com
In contrast, resistance to adefovir dipivoxil has been reported with mutations in the $\mathrm{D}$ and $\mathrm{B}$ domains of the enzyme. ${ }^{1,2}$ The mutations within the reverse transcriptase (rt) region, that were selected for during LAM therapy, have been designated as rtM204I/V/S (domain C) and rtL180M (domain B). ${ }^{1}$ The frequently detected, clinically important mutations that have been found to be associated with adefovir (ADV)-resistant HBV are designated as rtN236T (domain D) and rtA181T. ${ }^{2}$

LAM resistance has been shown to increase progressively during the treatment regimen, at a rate of $14 \%$ to $32 \%$ per year, reaching $70 \%$ after 48 months of treatment. ${ }^{3}$ Factors that have been reported to increase the risk of resistance include high levels of pre-therapy serum HBV DNA viral load, high levels of alanine aminotransferase and incomplete suppression of viral replication. $^{3,4}$ LAM resistance does not confer cross-resistance to adefovir dipivoxil. Mutations that confer LAM resistance have also been reported to decrease the in vitro sensitivity to the drug, by many folds. However, the rtM204I substitution has been detected in isolation, while rtM204V and rtM204S have been found only in association with other changes that take place in the B or A motifs.

Numerous other secondary modifications in the rt sequence have been documented in conjunction with $\mathrm{rtM} 204 \mathrm{~V} / \mathrm{I} / \mathrm{S}$, many of which are likely to be compensatory in nature. ${ }^{5}$ However, HBV resistance to ADV occurs less frequently ( $\sim 2 \%$ after the end of the 2 nd year of treatment) than resistance to LAM. ADV resistance is triggered by substitution of a threonine for an asparagine at codon 236 , which is located in the D motif of HBV; this has not been reported in the initial clinical trials, but was observed in two patients who had been shifted to the therapy upon development of resistance to LAM. ${ }^{2}$ Mutations in the viral polymerase gene are associated with the emergence of entecavir (ENT) resistance and were mapped to its domain B (rtS184G), domain C (rtS202I) and domain D (rtM250V).

With consideration to the above knowledge in the field, the present study was undertaken on a population of 75 Indian patients with HBV-related hepatocellular carcinoma (HCC) (Table 1). The current study findings represent additional data generated during whole genome analysis of the HBV genome infecting the studied population, which was the doctoral thesis of the first author of this manuscript.

\section{Materials and methods}

\section{Medication regimens}

Out of the 75 patients with HBV-related HCC, only 12 had a history of treatment with nucleoside/tide drugs. Of these, seven had 
Table 1. HBV-related HCC cases included in the study [w.r.t. medication]

\begin{tabular}{lll}
\hline Total Cases & Treated Nucleotide/sides & Untreated \\
\hline 75 & 12 & 65 \\
LAM & 7 & \\
$* *$ CHANGE IN MEDICATION & 3 (INITIALLY ON LAM/SHIFTED TO ENT) \\
ENT & 3 & \\
ADV & 2 & \\
CHANGE IN DRUG & 1 (INITIALLY ON ADV/SHIFTED TO ENT) \\
\hline
\end{tabular}

received LAM, three received ADV and two received ENT. Three of the patients on LAM were later shifted to ENT, and 1 on ADV initially was shifted to ENT.

HBV DNA was extracted by the standardized manual phenolchloroform method described, with slight modifications. The polymerase gene was amplified in a $25 \mathrm{~mL}$ PCR reaction mixture, containing $2 \mathrm{~mL}$ of the DNA sample, $10 \mathrm{mM}$ of each dNTP, $20 \mathrm{ng}$ of each primer and $1 \mathrm{U}$ of Taq DNA polymerase. Amplification was carried out in a thermal cycler (Biometra, Gottingen, Germany) for 30 cycles that were comprised of denaturation at $95^{\circ} \mathrm{C}$ for $60 \mathrm{sec}$, annealing at $67.5^{\circ} \mathrm{C}$ for $45 \mathrm{sec}$ and extension at $72^{\circ} \mathrm{C}$ for $45 \mathrm{sec}$, with a final extension step at $72^{\circ} \mathrm{C}$ for $10 \mathrm{~min}$. The above steps were accompanied by an initial denaturation at $95^{\circ} \mathrm{C}$ for $10 \mathrm{~min}$. The primer sequences used were 5'-CTGTAACACGAGAAGGGGTCCTAG-3' and 5'-GTGGGGTCACCATATTCTTGGG-3'. A 2-mL aliquot of the PCR products was resolved in a $2 \%$ agarose gel containing ethidium bromide and observed under UV light. The PCR products of interest were purified for sequencing using the Perfectprep Gel Clean-up Kit (Eppendorf, Westbury, NY, USA), as per the manufacturer's instructions. The PCR amplicons with a sharp band were sent for commercial sequencing, as per the guidelines of the company whose services were hired.

The PCR products for the HBV polymerase gene isolated from the HBV-related HCC cases were sequenced using the commercially available sequencing services from Macrogen, South Korea. The sequences obtained from Macrogen were submitted to Genbank under the accession number BankIt1600355: (61). The polymerase gene sequences were analyzed with the software http:// www.hepseq.org/Public/Tool/annotator_tool.php and the drugresistant mutations detected were studied in detail.

A total of 4 viz. (L80I (LAM), I169T (ENT), N236T (ADV) and A181V LAM/ADV)) mutations were observed in three patients. Two of the patients were harboring dual-drug resistant mutation. The drug-resistant mutants are shown in the Figures S1-S3, along with the results of statistical analysis.

Mutations such as the L80I observed in this study have been documented as associated with LAM resistance, and characterized as a compensatory mutation for M204V/I. ${ }^{6}$ They stated that the main M204V/I drug-resistant mutation and additional compensatory mutations such as $\mathrm{L} 80 \mathrm{I} / \mathrm{V}, \mathrm{V} 173 \mathrm{~L}$ and $\mathrm{L} 180 \mathrm{M}$ are associated with high-level resistance to LAM (3TC), telbivudine (L-dT) and emtricitabine (FTC). In addition, the importance of this particular mutation observed in our study needs to be further assessed since this novel mutation is not located in the supposed drug binding domain. The ability of rtL80I to mediate LAM resistance is of poignant general interest and deserves the utmost attention primarily for two reasons. First, the fact that mutations in the periphery of the nucleos(t)ide binding site may lead to resistance has to be taken into consideration when developing, applying and assessing resistance testing approaches. Second, it may also have an association with other drugs available for HBV treatment. ${ }^{7}$
Similarly, dual-drug-resistant mutations were observed in two of the HBV-related HCC cases in our study of patients from North India. The first case harbored the L80I and N236T mutations. L80I is a LAM mutation, while N236T is associated with ADV resistance. ${ }^{2}$ The main M204V/I drug-resistant mutation and the additional compensatory mutations-L80I/V, V173L and L180M-are associated with high-level resistance to LAM, L-dT and FTC. ${ }^{6}$ The other patient with dual-drug mutation was found to be harboring the I169T and A181V mutations. I169T has been associated with the ENT resistance mutations in LAM-resistant patients, and is believed to play a secondary role in ENT resistance; meanwhile, A181V/T is associated with resistance to ADV and/or LAM. ${ }^{2,7,8}$ These mutants were expected, as few of the HBV-related HCC cases in our study had been under nucleotide/nucleoside therapy in the past. The documentation of dual-drug mutants observed in the current study can be explained, however, as many patients (while on treatment) developed LAM mutations and were either switched to ADV or ENT.

This current report confirms the presence of previously reported drug-resistant mutations in the hepatitis B viral genome infecting a North Indian population. Its association with the advanced stage of the disease and to genotype $\mathrm{D}$, in particular, needs to be studied in depth. Although, drug resistance was not observed in the genome of HBV genotype $\mathrm{C}$, the polymerase gene of this genotype needs careful investigation as it has been reported to be more aggressive than the other genotype. ${ }^{9}$ The current study did not encounter cases with co-infection of the different genotypes of $\mathrm{HBV}$, as reported by Datta $\mathrm{S}$, et al, because most of the cases selected (50, data not shown) were from North and South India, where the predominant circulating HBV genotypes are D and A. ${ }^{9}$ However, the authors of this manuscript do not rule out the possibility of integration of HBV genomes in cases where genotype $\mathrm{C}$ has been reported (from Northeast India). Future prospective study on drug-resistant mutants, with respect to different genotypes of $\mathrm{HBV}$, will provide more information and may well provide scientific data in support of the school of belief that HBV genotype D is more prone to drug resistance. The present findings will also be of immense use for framing health programmes to fight against HBV infection in India, as the dominant genotype in this part of the globe is HBV genotype D.

\section{Acknowledgements}

The authors acknowledge financial assistance received from a University grant commission for completing the submitted work under a major research project.

\section{Conflict of interest}

The authors have no conflict of interests related to this publication. 


\section{Author contributions}

Carrying out the wet laboratory experiments (MPS), Guiding the scientific work \& sample collection (GA, PK), Helping with the bioinformatics part (SM, DB), framing the manuscript (MB).

\section{Supplementary information}

(Supplementary information is linked to the online version of the paper on the Exploratory Research and Hypothesis in Medicine website.)

\section{References}

[1] Stuyver LJ, Locarnini SA, Lok A, Richman DD, Carman WF, Dienstag JL, et al. Nomenclature for antiviral-resistant human hepatitis B virus mutations in the polymerase region. Hepatology 2001;33:751-757. doi:10.1053/ jhep.2001.22166.

[2] Angus P, Vaughan R, Xiong S, Yang H, Delaney W, Gibbs C, et al. Resistance to adefovir dipivoxil therapy associated with the selection of a novel mutation in the HBV polymerase. Gastroenterology 2003;125:292-297. doi:10.1016/
S0016-5085(03)00939-9.

[3] Lai CL, Chien RN, Leung NW, Chang TT, Guan R, Tai DI, et al. A one-year tria of lamivudine for chronic hepatitis B. Asia Hepatitis Lamivudine Study Group. N Engl J Med 1998;339:61-68. doi:10.1056/NEJM199807093390201.

[4] Leung N. Clinical experience with lamivudine. Semin Liver Dis 2002;22:1521. doi:10.1055/s-2002-35696.

[5] Delaney WE IV, Yang H, Westland CE, Das K, Arnold E, Gibbs CS, et al. The hepatitis B virus polymerase mutation $\mathrm{rtV} 173 \mathrm{~L}$ is selected during lamivudine therapy and enhances viral replication in vitro. J Virol 2003;7:11833-11841. doi:10.1128/JVI.77.21.11833-11841.2003.

[6] Ogata N, Fujii K, Takigawa S, Nomoto M, Ichida T, Asakura H. Novel patterns of amino acid mutations in the hepatitis B virus polymerase in association with resistance to lamivudine therapy in Japanese patients with chronic hepatitis B. J Med Virol 1999;59:270-276. doi:10.1002/(SICI)10969071(199911)59:3<270::AID-JMV2>3.0.CO;2-1.

[7] Tenney DJ, Levine SM, Rose RE, Walsh AW, Weinheimer SP, Discotto L, et al. Clinical emergence of entecavir-resistant hepatitis B virus requires additional substitutions in virus already resistant to Lamivudine. Antimicrob Agents Chemother 2004;48:3498-3507. doi:10.1128/AAC.48.9.3498-3507.2004.

[8] Yeh CT, Chien RN, Chu CM, Liaw YF. Clearance of the original hepatitis B virus YMDD-motif mutants with emergence of distinct lamivudine-resistant mutants during prolonged lamivudine therapy. Hepatology 2000;31:1318-1326. doi:10.1053/jhep.2000.7296.

[9] Datta S, Roychoudhury S, Ghosh A, Dasgupta D, Ghosh A, Chakraborty BC et al. Distinct distribution pattern of hepatitis B virus genotype C and D in liver tissue and serum of dual genotype infected liver cirrhosis and hepatocellular carcinoma patients. PLoS One 2014;9:e102573. doi:10.1371/journal. pone. 0102573 . 